\title{
ACCURACY VERSUS COMPLEXITY TRADE-OFF IN VAR MODELING: COULD TECHNICAL ANALYSIS BE A SOLUTION?
}

\author{
EVANGELOS VASILEIOU \\ University of the Aegean, School of Engineering \\ Department of Financial and Management Engineering \\ Kountouriotou 41, 82100 Chios, Greece \\ e.vasileiou@ba.aegean.gr
}

Received 22 February 2019

Accepted 3 September 2019

Published 22 October 2019

\begin{abstract}
Accurate Value at Risk (VaR) estimations are crucial for the robustness and stability of a financial system. Even though significant advances have been made in the field of risk modeling, many crises have emerged during the same period, and an explanation for this is that the advanced models are not widely applied in the financial industry due to their mathematical complexity. In contrast to the mathematically complex models that torture the data in the output stage, we suggest a new approach that filters the data inputs, based on Technical Analysis (TA) signals. When the trading signals suggest that the conditions are positive (negative) for investments we use data from the previously documented positive (negative) periods in order to calculate the VaR. In this way, we use input data that are more representative of the financial conditions under examination and thus VaR estimations are more accurate and more representative (nonprocyclical) than the conventional models' estimation that use the last nonfiltered $x$-day observations. Testing our assumptions in the US stock market for the period 2000-2017, the empirical data confirmed our hypothesis. Moreover, we suggest specific legislative adjustments that contribute to more accurate and representative VaR estimations: (i) an extra backtesting procedure at a lower than the $99 \%$ confidence level as a procyclicality test and (ii) to ease the minimum requirement of 250 observations that is currently the input threshold because it leads to less accurate VaR estimations.
\end{abstract}

Keywords: Value at risk; procyclicality; estimations accuracy; technical analysis; legislative framework.

JEL Codes: G15, G18, G24, G28, G32

\section{Introduction}

The advantages of a robust and stable financial system are widely known and documented in the financial literature (Levine 1997, Beck et al. 2000, Ang 2008,

This is an Open Access article published by World Scientific Publishing Company. It is distributed under the terms of the Creative Commons Attribution 4.0 (CC BY) License which permits use, distribution and reproduction in any medium, provided the original work is properly cited. 
Peia \& Roszbach 2015). The financial system's primary role is to efficiently allocate resources from savers to borrowers/enterprises in a way that maximizes welfare for everybody. In order for the intermediation procedure to work efficiently, the financial system should allow risk to be shared (Allen et al. 2004). Therefore, the importance of accurate risk estimation is crucial for a financial system's robustness, and for this reason several legislative initiatives have been adopted, i.e. the Committee of European Securities Regulators (CESR 2010), and the Basle Accords, which force asset management companies and banks, respectively, to measure and evaluate their financial risk on a daily basis.

The dominant measure for risk estimation is the well-known Value at Risk (VaR) which is defined as a statistical estimation of possible portfolio/instrument losses resulting from "normal" market movements. The probability of losses greater than the VaR is small ${ }^{\mathrm{a}}$ (Linsmeier \& Pearson 2000). We focus on the word "normal." What does the term "normal" really mean in this definition? Could the term normal be linked to a noncrisis period or to a future period exhibiting a pattern similar to the past? The second assumption is closer to the truth. Therefore, in this study, we assume that normality is linked to representativeness, and we define normality as cases where the upcoming performance is similar to the historical market behavior (data inputs) that we use to assess risk. Regulators and practitioners pay significant attention to VaR accuracy. As a result, there has been increased interest in VaR modeling among scholars.

The first VaR models that had been documented and applied, such as the Historical (HVaR), the Variance-Covariance (or Delta Normal, DNVaR) and the Monte Carlo Simulation (MCVaR), were econometrically simple. We call them "conventional" for the purposes of this study. These conventional models presented drawbacks regarding their reliability to accurately capture the real financial risk, especially when the financial trend changed (Hendricks 1996, Brooks \& Persand 2000).

However, the advances in econometrics and computer science enable scholars to fit the data and produce advanced, but complex, mathematical models that present accurate $\mathrm{VaR}$ estimations, even when the data inputs are inappropriate: extreme value theory (Assaf 2009), nonparametric Kernel Estimators (Yi-Hou Huang \& Tseng 2009), GARCH family models (Engle 2004, Angelidis et al. 2004, Stavroyiannis et al. 2012, Diamandis et al. 2011, Mabrouk \& Saadi 2012), Asymmetric Heterogeneous Autoregressive realized volatility model combined with Extreme Value Theory (Louzis et al. 2014), Markov Switching Regime (Billio \& Pelizzon 2000), Fuzzy VaR and Expected Shortfall models with elliptical distributions (Moussa et al. 2014), Extreme Learning Machine (Zhang et al. 2017), etc.

While studying the financial literature, the following question emerged: if the advanced models are as accurate as they promise, why have we had so many, and severe, crises in the last decades (Reinhart \& Rogoff 2008)? In theory, the VaR

\footnotetext{
a The legislative framework (CESR 2010) and the Basel Committee on Banking Supervision (2006) set the VaR estimations' accuracy at the $99 \%$ confidence level (in other words, there is $1 \%$ probability that the financial instrument/portfolio will fall in value more than the VaR estimation).
} 
models should capture the risk and in time inform all the participants that something is going to happen, and in such a case the crisis would not be so severe. In practice, this process appears to fail (crises emerge). Therefore, we conclude that either the advanced models are not accurate or that these models are not applied due to unspecified reasons. We certainly reject the former explanation that questions the advanced models, but we cannot reject the later assumption.

In this study, we assume that the advanced financial models are not really applied in the financial industry, and we present some examples that work toward validating our assumption. First, according to the legislative framework, a financial institution and/or an asset management company can adopt any model it wants for the VaR estimation, but in some specific paragraphs seems to recognize what really happens in financial industry. Particularly, CESR (2010, p. 26) and the Basel Committee on Banking Supervision (BCBS 2006, p. 115) mention that the models that are considered as the most popular in the financial industry are the simple and conventional models: the HVaR, DNVaR and MCVaR. Second, widely used financial systems, such as Bloomberg (PORT function), apply these conventional models to estimate VaR. The aforementioned are some strong indications that the industry applies conventional and simple models.

The next step in examining this issue was to try and understand why the available advanced models are not applied in the financial industry. Some scholars suggest that the mathematically advanced models are not applied in the financial industry because: (a) they are too complex to be understood, even by professional experts and (b) their application leads to increased costs that the financial companies try to avoid by taking advantage of deficiencies in the law. As far as the aforementioned, Jiménez-Martín et al. (2009) and Vasileiou (2016) present how risk analysts can manage the risk and avoid the legislative restrictions under the Basel and the CESR (2010), respectively. The "complexity issue" was perfectly described by Fama (1995) when he noticed that in some cases the contemporary financial economics models used so complex equations that nonmathematicians find them incomprehensible. Similarly, Ross (1993) described the advanced modeling as a procedure during which you torture the data long enough, in order to confess the crime. ${ }^{\mathrm{b}}$

Therefore, we figured out that in order for a model to be useful, it should be easy to understand and implement. As it has been mentioned above, a reason why the conventional and easily understood models provide inaccurate VaR estimations is that in many cases the data inputs used for estimating future financial risk are not appropriate (the "Garbage In, Garbage Out" issue as it is widely known in computer science). Conventional VaR models are not accurate especially when the financial trend changes (Hendricks 1996, Brooks \& Persand 2000), and their estimations lead to procyclicality (Youngman 2009, Adrian \& Shin 2013), which destabilizes the financial system. (Vasileiou 2017) suggests that even the simplest VaR models could present significantly more accurate $\mathrm{VaR}$ estimations than their conventional

\footnotetext{
${ }^{\mathrm{b}}$ This phrase belongs to a friend of Ross as he mentions in his study.
} 
forms when the data inputs are more representative of the examined financial risk conditions.

Therefore, in this study, we filter the data inputs in order to improve the accuracy of the conventional models. We use Technical Analysis (TA) rules as the data filtering criterion. The main idea is that if TA rules accurately indicate the financial market's trends, they can contribute to the estimation process and show us the past observations that are more representative of the examined financial conditions. The TA filtering model does not use the last $x$-day observations as data inputs, as the conventional models do, but filters the data and uses the last $x$-day observations that are similar (according to the TA signals) to the examined period. By adding the data filtering procedure into the $\mathrm{VaR}$ estimation process, we try to resolve or at least to reduce the Garbage In, Garbage Out issue. If the examined models empirically present more accurate VaR estimations, we will show that in many cases it is not the models that are inaccurate, but the inappropriate data that make the models appear unsuccessful.

We test our assumption using the easy to apply and to communicate HVaR model in its conventional version $(\mathrm{CHVaR})$ and we show that the newly suggested approach significantly improves $\mathrm{VaR}$ estimation accuracy, without using complex mathematical procedures. In order to provide empirical evidence, we examine the world's most popular stock index, the S\&P 500 (SPX). The empirical evidence shows that even the simplest TA rules could prove beneficial and contribute to more accurate $\mathrm{VaR}$ estimations. In addition, this study suggests some easy to apply modifications regarding the VaR legislative framework that may lead to more accurate estimations and may reduce procyclicality in conventional VaR models.

As to the structure of this paper, it goes as follows: Sec. 2 analyzes the theoretical framework of the newly suggested approach, and Sec. 3 examines the US stock market and provides empirical evidence that this novel approach presents fewer overshootings $^{\mathrm{c}}$ and more representative $\mathrm{VaR}$ estimations than the conventional one. Section 4 discusses further advantages of the newly suggested approach, and Sec. 5 concludes the study.

\section{Theoretical Framework of the Technical Analysis Historical VaR}

In this study, we try to present an easy to apply approach that improves a VaR model's estimations accuracy. Our intent is to focus on the methodological innovation that we want to present; therefore, we use simple VaR models and simple TA rules in order to not disorient the reader with complex mathematical models. ${ }^{\mathrm{d}}$

\footnotetext{
${ }^{\mathrm{c}}$ An overshooting is a 1-day change in the portfolio's value that exceeds the related 1-day value-at-risk measure calculated by the model (CESR 2010).

$\mathrm{d}$ An additional benefit of this intention is that anyone who is interested to apply the newly suggested model can easily obtain the results because in the financial industry computational time is as significant a factor as complexity (see Pritsker 1997 for the tradeoff between accuracy and the computational time issue). The methods we suggest in this paper do not require complex and time-consuming procedures.
} 
Moreover, we want to present a model that could be easily applied by anyone, so the simplest trading rules and VaR models are combined for the scope of this paper.

\subsection{The conventional historical VaR model: Drawbacks and ideas for the improvement of its estimations' accuracy}

We use the CHVaR model, according to which the VaR estimation is the worst $p$-percentile of the last $x$-day trading returns. The legislative framework suggests that the data observation period should not be less than 1-year data observations (approx. 250-trading days $(x \geq 250))^{\mathrm{e}}$ and that the confidence level (c.l.) should be set at $99 \%(p=$ c.l. $=99 \%)($ BCBS 1995, CESR 2010).

The CHVaR model may provide accurate estimations when the upcoming period is similar to the past $x$-day observations that were used as input, but if the $x$-day period is not defined correctly, the VaR estimations will be inaccurate (Hendricks 1996, Brooks \& Persand 2000). Additionally, in the latter case the CHVaR model does not accurately incorporate the financial trend changes and this leads to procyclicality (Vasileiou \& Pantos 2018). Therefore, the CHVaR model presents inaccurate and procyclical VaR estimations, not strictly due to the unsuitability of the model, but rather due to inappropriate data inputs. In this study, we do not want to torture the data by using a complex output formula; we choose to filter the input data and use only the most appropriate/representative past observations in order to achieve more accurate and more representative VaR estimations. TA could be a very useful tool in establishing a data filtering procedure which enables us to reduce the well-known Garbage In, Garbage Out issue.

\subsection{Technical analysis (TA): Conventional arguments, and its new role in the VaR estimation process}

TA is a sum of applied practical rules that suggest to the investors that long (or short or zero) positions should be taken when specific signals are observed. These rules are based on historical prices/data, and try to forecast or to confirm the financial trend. A "buy signal" is an indication for growth period, while a "sell signal" is linked to recession/crisis periods. ${ }^{f}$

Most of the times, in the financial literature TA usage is limited only to the profitability issue and whether the TA could outperform a Buy-and-Hold ( $\mathrm{BnH})$ strategy, without examining any further benefits that TA may have to offer. Studying the respective literature we observed that in these arguments the

\footnotetext{
e There are some ways to overcome the specific limitation, such as by adopting a weighted model (e.g. Exponential Weighted Moving Average (EWMA) model). Therefore, why does the law define an effective period as longer than a year? In most of the cases, a 250-observation data period is applied, and that is the reason we present our assumptions for the 1-year data sample. In Sec. 4, we quantitatively show that the specific limitation does not help to promote financial stability and robustness. On the contrary, laxity of regulation may be beneficial.

f Nazário et al. (2017) present an extensive analysis of the empirical literature review of TA, which shows the methodological advances and the importance of TA.
} 
transaction costs played a crucial role, and we highlighted a characteristic example: Fama \& Blume (1966) suggest that TA fails to outperform the BnH strategy in the late 1950s after adjusting for transaction costs; however, Sweeney (1988) questions their findings examining the same sample of stocks for a later period (1970-1982) including ". . .low but feasible transactions costs." Their counterargument triggered the following question: why should TA benefits be limited only to profitability and outperformance issues? If TA fails to outperform the $\mathrm{BnH}$ strategy due to the transaction costs, this does not mean that the signals are not accurate, and the accurate signals should not be ignored.

In this study, when the signals correctly suggest that a recession/growth period has come, we give them a "second chance" to be beneficial in other fields of financial analysis, such as the VaR. If the technical signals are accurate, they will be a good indication of how the market behaved during previous days when conditions were similar (favorable or unfavorable for investments). This way the historical approach is really historical and representative of the examined financial conditions because it presents the worst $p$-percentile of the last $x$-days when the market experienced similar financial conditions (positive or negative trend).

There are several TA rules, such as Simple Moving Average (SMA), Exponential Moving Average (EMA), RSI Index, MACD, Stochastic rules, etc. which may be used in order to outperform the stock market's performance versus the BnH, and that could be good filtering indicators for the scope of this study. However, as we mentioned at the beginning of this section, because of our focus on simplicity and methodology, the simplest trading rules are adopted: the SMA rules. ${ }^{\mathrm{g}}$

We adopt the suggestion of Ülkü \& Prodan (2013) and when current closing price $\left(P_{t}\right)$ crosses the $x$-simple moving average $\mathrm{SMA}_{l}$ from below, this is an indication that the market trend is positive, as long as $P_{t}>\mathrm{SMA}_{l t}$, where $l=$ the lag-length of the trading rule. When the opposite indications are observed, a negative trend is suggested. Quantitatively, $\mathrm{SMA}_{l}$ is defined as follows:

$$
\mathrm{SMA}_{l}=\frac{\sum_{t=1}^{l} P_{t}}{l},
$$

where $t=$ the examined day, $l=$ the lag-length (number of previous close prices) for which the moving average is calculated. SMA rules confirm that the financial trend is positive or negative, and our data filtering procedure is based on these rules' indications/signals.

\subsection{VaR and TA: The theoretical framework of the newly suggested model}

In this study, we present an implementation of the TA HVaR, which is based on the easy to apply and understand CHVaR. Our goal is to increase estimation accuracy 

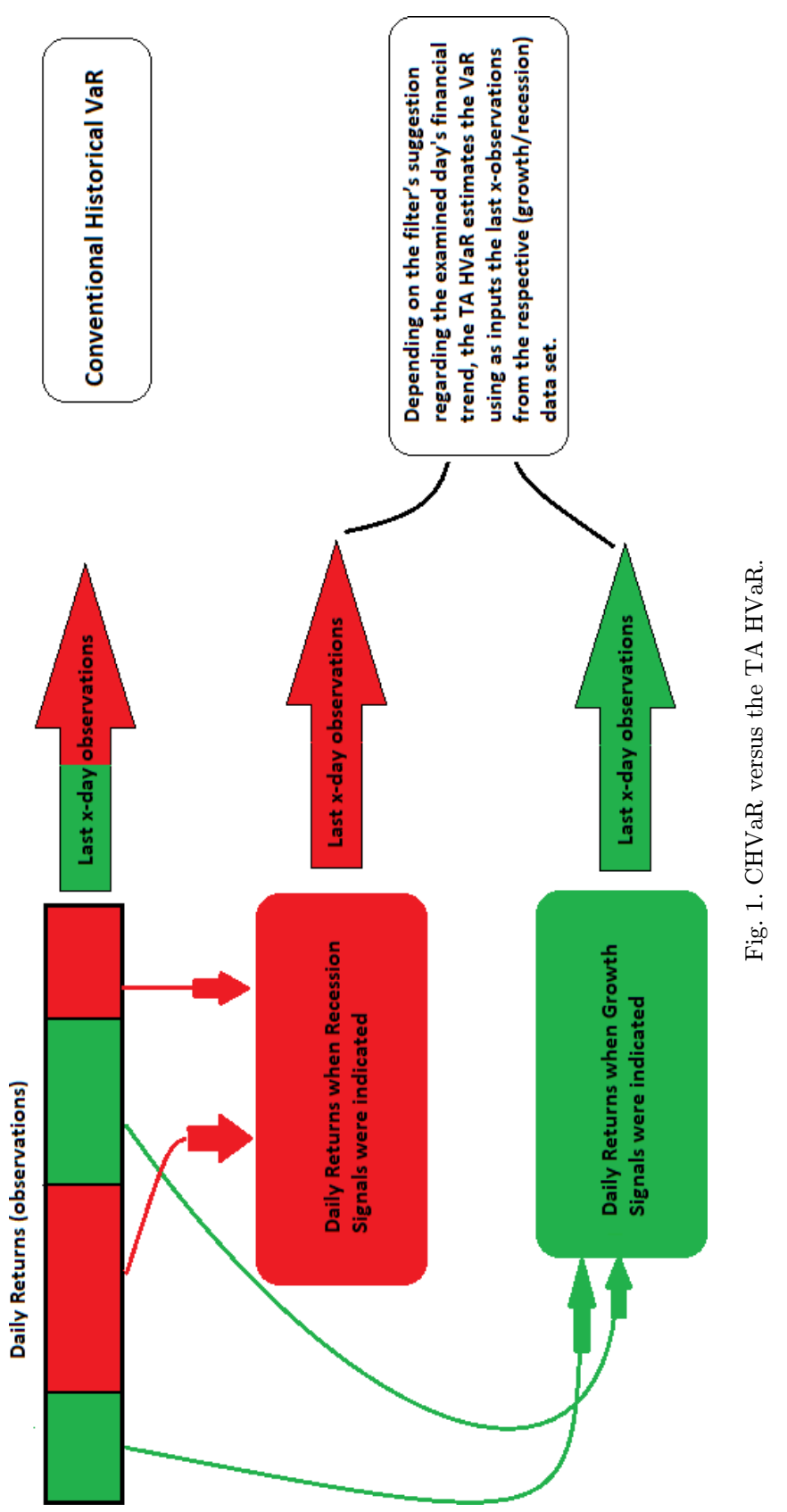


\section{E. Vasileiou}

by incorporating the technical signals as a data filtering indicator into the conventional estimation procedure. Particularly, we assume that investors exhibit a different behavior when the stock market is in a recession period than when the stock market is in a growth period. Therefore, we set the historical data in two regimes, growth and recession, and depending on the regime that the current financial conditions belong to, we use data from different datasets (regimes) in order to estimate the VaR

- when the trading signal suggests that the financial market is in growth period (buy signal), the $\mathrm{VaR}$ is estimated by the $p$-percentile of last $x$-observations of the previously documented growth periods and

- when the SMA rule suggests that the financial market's trend is negative (sell signal), the data observations that are used for the VaR estimations are the last $x$-day observations that were documented during negative trends.

This way the TA HVaR model will provide more accurate and more representative VaR estimations than in its conventional form $(\mathrm{CHVaR})$, because its data inputs are similar to the examined period. Filtering the data inputs, the CHVaR turns to the TA HVaR model, and the estimations become more historical because the model uses past observations that more accurately reflect the risk conditions under examination.

Figure 1 graphically presents the theoretical difference between the CHVaR and the TA HVaR model. The former uses as inputs the last $x$-day observations, and the later depending on the filter's suggestion regarding the examined day's financial trend, estimates the VaR using as inputs the last $x$-observations from the respective (growth/recession) data set. This way, the TA HVaR model's estimations should be more representative of the examined financial conditions than the CHVaR model.

\section{Empirical Evidence}

In order to empirically show that our assumptions are valid we test them using as sample the US stock market, and particularly the SPX index for the period 20002017 which includes at least two major crisis periods: (i) the dot.com crisis and (ii) the subprime mortgage crisis 2007-2009.

Table 1 presents the summary statistics of the SPX daily returns during the examined period. The Jarque-Bera test shows that the distribution is not normal, which is an indicator that a trading strategy may be profitable (Neftci 1991); therefore, for the purpose of this study it means that the SMA signals may be accurate.

Figure 2 graphically presents an additional view of the theoretical framework of our study using real data from our sample. Panel (a) shows the stock market's performance during the examined period. The procyclicality issue and the inaccurate estimations during changes in financial trends are graphically presented in spots $\mathrm{A}$ and B. Before spot A, there is a long-term period of growth, but a crisis emerges. 
Table 1. Descriptive statistics of SPX index daily returns (2000-2017).

\begin{tabular}{lc}
\hline & SPX daily returns \\
\hline Mean & $0.021 \%$ \\
Median & $0.049 \%$ \\
Maximum & $11.58 \%$ \\
Minimum & $-9.04 \%$ \\
Std. dev. & $1.21 \%$ \\
Skewness & -0.0103 \\
Kurtosis & 11.85 \\
Jarque-Bera (probability) & $14787.26(0.00 \%)$ \\
Observations & 4531 \\
\hline
\end{tabular}

When a CHVaR model is applied, the last 250-day observations lead to VaR estimations that may underestimate the risk. On the other hand, in spot B, when the stock market passes from the recession/crisis period to a growth period the $\mathrm{CHVaR}$ model may overestimate the risk, because the data inputs that are used for the VaR estimation come from the crisis period. ${ }^{\mathrm{h}}$ Such VaR estimations lead to procyclicality.

Panel (b) presents the SPX and the 20-, 125- and the 250-day SMAs. ${ }^{i}$ We observe that the shorter term $\mathrm{SMA}_{20}$ is very close to the stock market's index performance; however, as Panel (c) shows, its signals are not clear enough because the rule suggests constant regime/trend changes (higher and lower than zero values). ${ }^{j}$

The next step of our analysis is to quantitatively show whether the SMAs rules provide us with accurate signals regarding the financial trend. A quantitative indication that the SMAs rules accurately confirm the financial trends could be the profitability of a trading strategy which is based on these rules, when we do not take into consideration the transaction costs. The TA strategy follows these simple rules: (i) when the trading signal suggests that there is a growth period, we take long positions equal to the index and (ii) when the trading signal confirms the negative trend, we sell all the securities.

The BnH Strategy during the examined period performed $81.97 \%$. The results of the abovementioned trading strategy which is based on the 20-, 125- and 250-day SMA are $-10.17 \%, 25.92 \%$ and $87.94 \%$, respectively (Table 2). Another indication

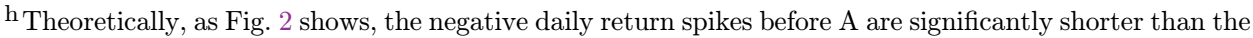
upcoming losses, and this means that the CHVaR model will underestimate the risk. In spot B, the previous negative spikes are significantly longer than the negative spikes in the upcoming period. This means that the CHVaR model in spot B may overestimate the risk. Moreover, Fig. 2 shows that in spot A the model should in time inform us of the upcoming crisis, but, on the contrary, it indicates a VaR lower than the respective $\mathrm{VaR}$ in spot $\mathrm{B}$ where the crisis has ended. This is an indication for procyclicality. Below we quantitatively confirm the aforementioned.

${ }^{\mathrm{i}}$ We present indicatively the monthly (20-day), the semiannual (125-day), and the 1-year (250-day) SMA, in order to include in our empirical findings shot-, med- and long-term SMA rules.

${ }^{\mathrm{j}}$ Indicatively, we observe that during the 2007-2008 crisis the $\mathrm{SMA}_{20}$ rule is often in the positive regime. On the contrary, the $\mathrm{SMA}_{125}$ and $\mathrm{SMA}_{250}$ are almost always in negative regime, and change to a positive regime in 2009.
} 


\section{E. Vasileiou}

regarding the appropriateness of the SMA rule and the adopted lag-length, is to examine the correlations between SPX Index of the next day $\left(\mathrm{SPX}_{t+1}\right)$ and the 20-, 125- and the 250-day SMA rules $\left(P_{t}-\mathrm{SMA}_{l t}\right)$. In theory, the higher the correlations, the more representative the SMA rules for the examined financial conditions. For the SPX and during the examined period the aforementioned correlations are $17.55 \%$,

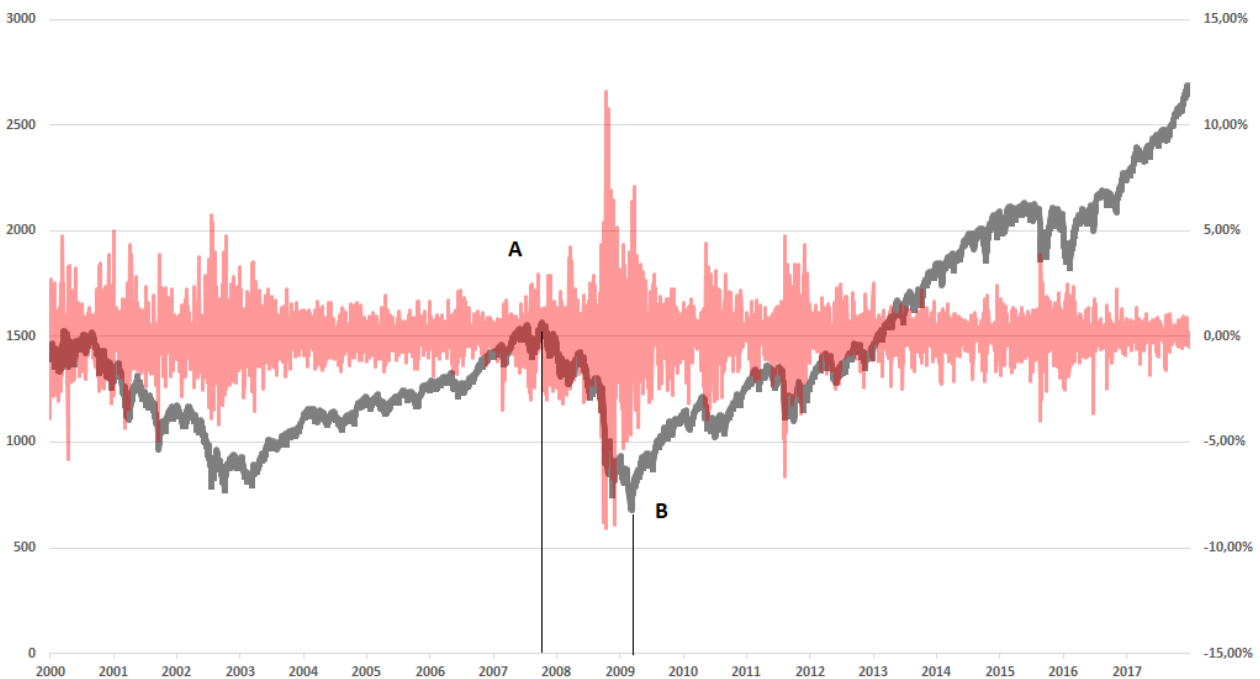

(a)

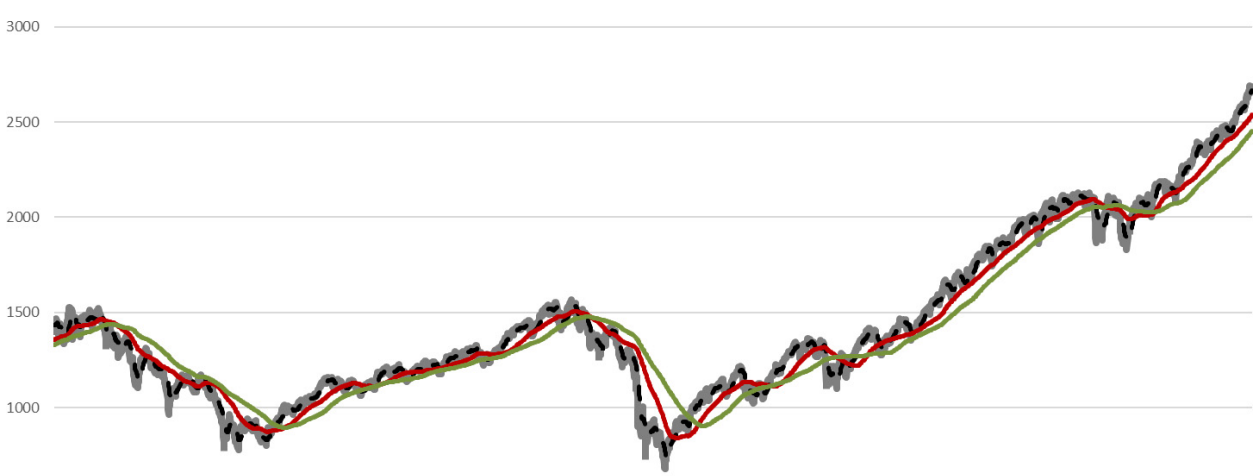

500

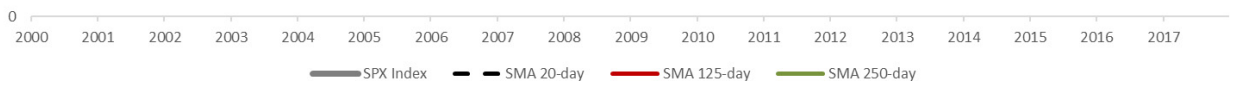

(b)

Fig. 2. SPX index performance and TA. (a) SPX performance and daily returns 2000-2017, (b) SPX performance and the 20-, 125- and the 250-day Simple Moving Average (SMA) 2000-2017 and (c) SPX SMA rule's signal 2000-2017 $\left(P_{t}-\mathrm{SMA}_{l t}\right)$. 


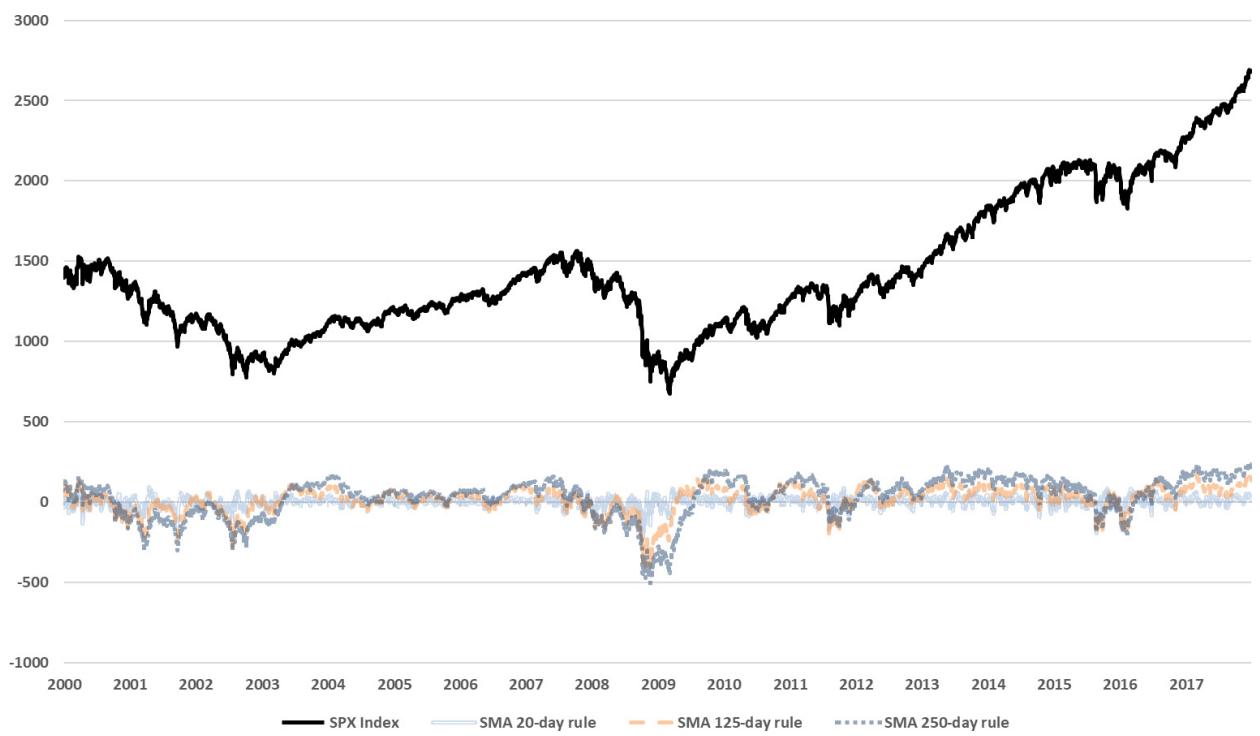

(c)

Fig. 2. (Continued)

Table 2. SPX and SMA rules performance during 2000-2017 period.

\begin{tabular}{lcccc}
\hline & $\mathrm{BnH}(\%)$ & $\mathrm{SMA}_{20}(\%)$ & $\mathrm{SMA}_{125}(\%)$ & $\mathrm{SMA}_{250}(\%)$ \\
\hline Returns & 81.97 & -10.97 & 25.92 & 87.94 \\
SPX & where $t=$ the current day and \\
$\quad$ & - & 17.55 & 40.47 & 52.28 \\
$\quad l=$ the lag-length. & & & & \\
\hline
\end{tabular}

$40.47 \%$ and $52.28 \%$, respectively (Table 2), which is another indication that the $\mathrm{SMA}_{20}$ may not be the most appropriate filtering criterion.

Therefore, the results of Table 2 quantitatively show that for the US case and for the examined SMAs the longer-term trading rules present more accurate signals than the shorter-term because if we adopt the abovementioned strategy, the returns are more profitable and the SMA rule more correlated/representative of the financial conditions. Theoretically, for our study's purpose these statistics indicate that the longer-term SMAs of our sample may be more appropriate data filtering indicators as they are better at accurately capturing market trend changes and are also more representative of the financial market's conditions.

Table 3 empirically shows the results taking into consideration the current legislative framework (at least 250-day historical data, 99\% confidence level (c.l.)). In our study, concerning the number of observations used for the VaR estimations, we present the case of the 250-day historical data, because it is more accurate than the 


\section{E. Vasileiou}

Table 3. CHVaR model versus $\mathrm{SMA}_{t}$ TA HVaR models, where $l=20,125$ and 250 days using 250 observations at the $99 \%$ confidence level.

\begin{tabular}{|c|c|c|c|c|}
\hline & CHVaR & $\begin{array}{l}\mathrm{SMA}_{20} \mathrm{TA} \\
\mathrm{HVaR}\end{array}$ & $\begin{array}{l}\mathrm{SMA}_{125} \mathrm{TA} \\
\text { HVaR }\end{array}$ & $\begin{array}{l}\mathrm{SMA}_{250} \mathrm{TA} \\
\mathrm{HVaR}\end{array}$ \\
\hline \multicolumn{5}{|c|}{ Panel A. Descriptive statistics of the CHVaR and the $\mathrm{SMA}_{l}$ TA HVaR models for the SPX (2000-2017) } \\
\hline Violations & 51 & 59 & 53 & 48 \\
\hline Violations \% & $1.13 \%$ & $1.30 \%$ & $1.17 \%$ & $1.06 \%$ \\
\hline Mean & $-3.10 \%$ & $-3.17 \%$ & $-3.06 \%$ & $-3.03 \%$ \\
\hline SD & $1.64 \%$ & $1.63 \%$ & $1.64 \%$ & $1.64 \%$ \\
\hline Min & $-8.86 \%$ & $-8.19 \%$ & $-8.86 \%$ & $-8.86 \%$ \\
\hline $\operatorname{Max}$ & $-1.39 \%$ & $-1.28 \%$ & $-1.28 \%$ & $-1.39 \%$ \\
\hline MSE & $0.139 \%$ & $0.144 \%$ & $0.136 \%$ & $0.135 \%$ \\
\hline $\begin{array}{l}\text { Deviations when overshootings } \\
\text { are observed }\end{array}$ & $-0.822 \%$ & $-0.716 \%$ & $-0.688 \%$ & $-0.755 \%$ \\
\hline \multicolumn{5}{|c|}{ Panel B. Backtesting results of the CHVaR and the $\mathrm{SMA}_{l}$ TA HVaR models for the SPX (2000-2017) } \\
\hline 2000 & 4 & 4 & 5 & 5 \\
\hline 2001 & 2 & 2 & 3 & 2 \\
\hline 2002 & 3 & 2 & 3 & 3 \\
\hline 2003 & 0 & 1 & 1 & 0 \\
\hline 2004 & 1 & 0 & 0 & 1 \\
\hline 2005 & 3 & 1 & 1 & 2 \\
\hline 2006 & 3 & 3 & 3 & 3 \\
\hline 2007 & 8 & 12 & 8 & 7 \\
\hline 2008 & 10 & 15 & 15 & 10 \\
\hline 2009 & 0 & 0 & 1 & 1 \\
\hline 2010 & 3 & 0 & 2 & 4 \\
\hline 2011 & 4 & 5 & 3 & 2 \\
\hline 2012 & 1 & 0 & 0 & 0 \\
\hline 2013 & 1 & 2 & 2 & 1 \\
\hline 2014 & 2 & 2 & 2 & 2 \\
\hline 2015 & 5 & 6 & 2 & 3 \\
\hline 2016 & 1 & 4 & 2 & 2 \\
\hline 2017 & 0 & 0 & 0 & 0 \\
\hline Sum of violations & 51 & 59 & 53 & 48 \\
\hline
\end{tabular}

CHVaR models that use more observations. ${ }^{\mathrm{k}}$ Table 3 presents the results of the CHVaR approach and the newly suggested TA HVaR approach using as a filtering indicator the 20-, 125- and the 250-day SMA rule. The $\mathrm{SMA}_{20}$ rule is not beneficial for more accurate than the conventional VaR estimations, because it fails to capture the financial trend. On the other hand, the results show that, the $\mathrm{SMA}_{250}$ and $\mathrm{SMA}_{125}$ TA HVaR models are beneficial ${ }^{\mathrm{l}}$ and present more representative of the real

\footnotetext{
${ }^{\mathrm{k}}$ We tested the 500-day, 750-day, 1000-day and the 1250-day CHVaR models but the 250-day CHVaR model was the most accurate and representative of the real financial risk (these data are available upon request).

${ }^{1}$ The findings in Table 2 validate our abovementioned assumption that the longer-term SMA rules $\left(\mathrm{SMA}_{125}\right.$ and $\left.\mathrm{SMA}_{250}\right)$ could be more beneficial than the shorter-term $\left(\mathrm{SMA}_{20}\right)$.
} 
financial conditions $\mathrm{VaR}$ estimations than the $\mathrm{CHVaR}$, because the $\mathrm{SMA}_{250}$ and $\mathrm{SMA}_{125}$ TA HVaR (hereafter the term TA HVaR models refers to them) models:

- present lower Mean Square Error (MSE) relative to the daily returns than the CHVaR models and

- when overshootings are documented these deviations are narrower (Panel A), and this is achieved without presenting more conservative VaR estimations (means of TA VaR estimations are lower than the mean $\mathrm{CHVaR}$ ), which is an indication for more representative and less procyclical VaR estimations.

However, according to the four overshootings rule ${ }^{\mathrm{m}}$ of the legislative framework, the TA VaR models do not improve the accuracy, because the specific threshold is violated on three occasions, similar to the CHVaR case (Panel B).

Therefore, taking into consideration the above-mentioned, we could suggest that the adjustment of the appropriate trading rule, in our case the $\mathrm{SMA}_{125}$ and $\mathrm{SMA}_{250}$ rules, significantly improved the representativeness of VaR estimations. However, strictly according to the legislative framework the TA adjustment has no added value, because only the four-violation rule is examined. ${ }^{\mathrm{n}}$ In such a case, an investment company/bank may be unmotivated to adopt a better than the CHVaR model (e.g. $\mathrm{SMA}_{250}$ or $\mathrm{SMA}_{125}$ TA HVaR).

This is a characteristic case in which the procyclicality issue/risk is hidden. The legislative framework does not examine whether the VaR estimations are representative of the financial risk. It examines only the number of violations per year (250 observations) at the $99 \%$ c.l. This backtesting procedure enables us to conclude whether a model underestimates the risk (more than four overshootings), but it does not examine whether the tested model overestimates the risk (CESR 2010, BCBS 1996). ${ }^{\circ}$ How could we overcome this problem?

Among the scientifically accepted models, the binomial Kupiec test (1995) presents similarities to the financial market's backtesting procedures because it examines only the number of overshootings. Therefore, the Kupiec test at a lower than the $99 \%$ c.l. could be a solution for testing a model's ability to representatively estimate the risk, because it enables us to examine not only the underestimation of risk, but

\footnotetext{
$\mathrm{m}$ According to CESR (2010) if the number of overshootings for the most recent 250 business days exceeds 4 at the $99 \%$ c.l. the competent authority may take measures and apply stricter criteria to the use of VaR. Similarly, in BCBS (2006) the Green Zone (up to 4 overshooting per 250 observations) "corresponds to backtesting results that do not themselves suggest a problem with the quality or accuracy of a bank's model." More than four overshootings mean increases in the multiplication factor applicable to the internal model's capital requirement. Therefore, four overshootings is the legislative threshold that is used to determine the accuracy of a VaR model and for this reason we call it the four overshootings rule.

$\mathrm{n}$ The law does not examine the deviation when a VaR violation is presented. This policy does not promote the need for more representative $\mathrm{VaR}$ estimations, e.g. if the daily losses are $2 \%$ and model $\mathrm{A}$ has an estimation $\mathrm{VaR}_{\mathrm{A}}=1.98 \%$ and model $\mathrm{B}, \mathrm{VaR}_{\mathrm{B}}=1 \%$, both models have one violation. However, qualitatively model $\mathrm{B}$ is more representative.

${ }^{\circ}$ The overshootings range is $0-4$. More than four overshootings indicate that the model underestimates the risk, but what happens when the model overestimates the risk? The down-limit is zero, and the number of overshootings cannot be a negative number.
} 


\section{E. Vasileiou}

Table 4. CHVaR model versus $\mathrm{SMA}_{l}$ TA HVaR models, where $l=20,125$ and 250 days using 250 observations at the $97.5 \%$ confidence level.

\begin{tabular}{lcccc}
\hline & & $\mathrm{SMA}_{20} \mathrm{TA}$ & $\mathrm{SMA}_{125} \mathrm{TA}$ & $\mathrm{SMA}_{250} \mathrm{TA}$ \\
& $\mathrm{CHVaR}$ & $\mathrm{HVaR}$ & $\mathrm{HVaR}$ & $\mathrm{HVaR}$ \\
\hline Panel A. Descriptive statistics of the CHVaR and the SMA & TA HVaR models for the SPX & $(2000-2017)$ \\
Violations & $\mathbf{1 3 0}$ & $\mathbf{1 2 6}$ & $\mathbf{1 1 9}$ & $\mathbf{1 2 5}$ \\
Violations (\%) & 2.87 & 2.78 & 2.63 & 2.76 \\
Mean & $-2.33 \%$ & $-2.41 \%$ & $-2.33 \%$ & $-2.30 \%$ \\
SD & $1.14 \%$ & $1.14 \%$ & $1.16 \%$ & $1.16 \%$ \\
Min & $-6.11 \%$ & $-6.00 \%$ & $-6.11 \%$ & $-6.51 \%$ \\
Max & $-0.85 \%$ & $-0.86 \%$ & $-0.85 \%$ & $-0.85 \%$ \\
MSE & $0.083 \%$ & $0.087 \%$ & $0.084 \%$ & $0.082 \%$ \\
Deviations when overshootings are observed & $-0.784 \%$ & $-0.789 \%$ & $-0.767 \%$ & $-0.731 \%$ \\
Panel B. Backtesting results of the CHVaR and & the SMA & TA HVaR models for the SPX & $(2000-2017)$ \\
2000 & 8 & 8 & 8 & 11 \\
2001 & 6 & 7 & 4 & 5 \\
2002 & 13 & 12 & 11 & 11 \\
2003 & 1 & 2 & 2 & 2 \\
2004 & 4 & 0 & 2 & 4 \\
2005 & 5 & 2 & 2 & 3 \\
2006 & 7 & 7 & 6 & 7 \\
2007 & 16 & 18 & 17 & 15 \\
2008 & 21 & 25 & 25 & 21 \\
2009 & 0 & 3 & 4 & 2 \\
2010 & 5 & 2 & 3 & 6 \\
2011 & 12 & 11 & 6 & 6 \\
2012 & 1 & 0 & 2 & 3 \\
2013 & 4 & 2 & 4 & 4 \\
2014 & 9 & 2 & 8 & 9 \\
2015 & 9 & 16 & 8 & 8 \\
2016 & 4 & 7 & 3 & 3 \\
2017 & 5 & 2 & 4 & 5 \\
Sum of violations & $\mathbf{1 3 0}$ & $\mathbf{1 2 6}$ & $\mathbf{1 1 9}$ & $\mathbf{1 2 5}$ \\
\hline & & & &
\end{tabular}

the overestimation also. Table 4 has a similar structure as Table 3, but it examines the VaR estimations at the $97.5 \%$ c.l., ${ }^{\mathrm{p}}$ for which the accepted overshootings range according to the Kupiec test is between 2 and 11. Fewer than 2 overshootings per year mean that the model overestimates the risk, and higher than 11 mean that the model underestimates the risk.

The results show that at the $97.5 \%$ c.l. the CHVaR model is considered inappropriate in 7 out of the 18 examined years: in four cases/years due to risk

PIn some papers, VaR is examined at the $95 \%$ c.l., but we choose to present the $97.5 \%$ c.l., because the legislative framework examines the risk too conservatively: (a) it examines the VaR at the most conservative c.l. (99\%) and (b) the number of accepted violations per the 250 last observations is lower than the threshold that the Kupiec (1995) test suggests (4 vs 5, respectively). Therefore, we try to be conservative in our estimations, but at the same time we wish to offer suggestions on how to examine and resolve the procyclicality issue. 
underestimation (2002, 2007, 2008 and 2011), and in 3 due to risk overestimation (2002, 2009 and 2012). It is notable that 1 year the model underestimates the VaR (2002, 2008 and 2011), and in the next year it overestimates the VaR $(2003,2009$ and 2012, respectively). These results are evidence of procyclicality, ${ }^{\mathrm{q}}$ and confirm Brooks \& Persand (2000) that conventional VaR models are inaccurate when the financial trends change.

The contribution of the $\mathrm{SMA}_{250}$ and $\mathrm{SMA}_{125}$ rules in the newly suggested TA $\mathrm{HVaR}$ procedure is that they made the VaR estimations more representative of the real financial conditions when compared to the $\mathrm{CHVaR}$ model and they reduced the procyclicality with the data filtering procedure. Particularly, as the results show these TA HVaR models are considered inaccurate only in the 2007-2008 period during which they underestimated the risk, while they did not overestimate the risk in any year during the examined period 2000-2017. This means that after crises the TA HVaR models did not overestimate the risk as the CHVaR model did, so they did not cause procyclicality. When the market had good prospects for recovery/growth the TA HVaR models gave a signal for risk taking (compare CHVaR and TA HVaR models in 2002-2003, 2008-2009 and 2011-2012, Table 4, Panel B).

Therefore, even if CHVaR and TA HVaR models were equally accurate at the $99 \%$ c.l., at the $97.5 \%$ c.l. the results show that the $\mathrm{SMA}_{125}$ and $\mathrm{SMA}_{250}$ TA HVaR are significantly more reliable than the CHVaR models because they are significantly more representative without presenting procyclicality effects. Figure 3 graphically presents the specific case: the comparison between models CHVaR and $\mathrm{SMA}_{250} \mathrm{TA}$ HVaR. In Panel (a), we present the VaR comparison of the CHVaR and the $\mathrm{SMA}_{250}$ TA HVaR model at the $99 \%$ c.l. relative to the SPX performance, and in Panel (b), we present the same variables at the $97.5 \%$. The figures show the CHVaR model, which is not flexible enough to incorporate financial trend changes, becomes more flexible when the trading rules filtering procedure is incorporated to the VaR estimation: the spikes during recession periods and the reduction of the VaR during growth periods show these regime changes. The increased flexibility due to the TA adjustment on the HVaR model makes the VaR estimations more representative of the current financial risk conditions and reduces almost completely the procyclicality (Tables 3 and 4 provide the empirical evidence). Therefore, it is not always the models that are inaccurate, but in many cases it is the inappropriate data that make the models appear unsuccessful.

\section{Further Advantages of the Newly-Suggested Approach}

We should highlight that this study presents a simple implementation of the specific idea in order to enable the readers to focus on the theoretical framework and to avoid the complexity issues. However, it is important to note that the results could be

qThe results during the period 2008-2009 validate our assumptions for procyclical VaR estimations, which we analytically present in Fig. 2(a). 


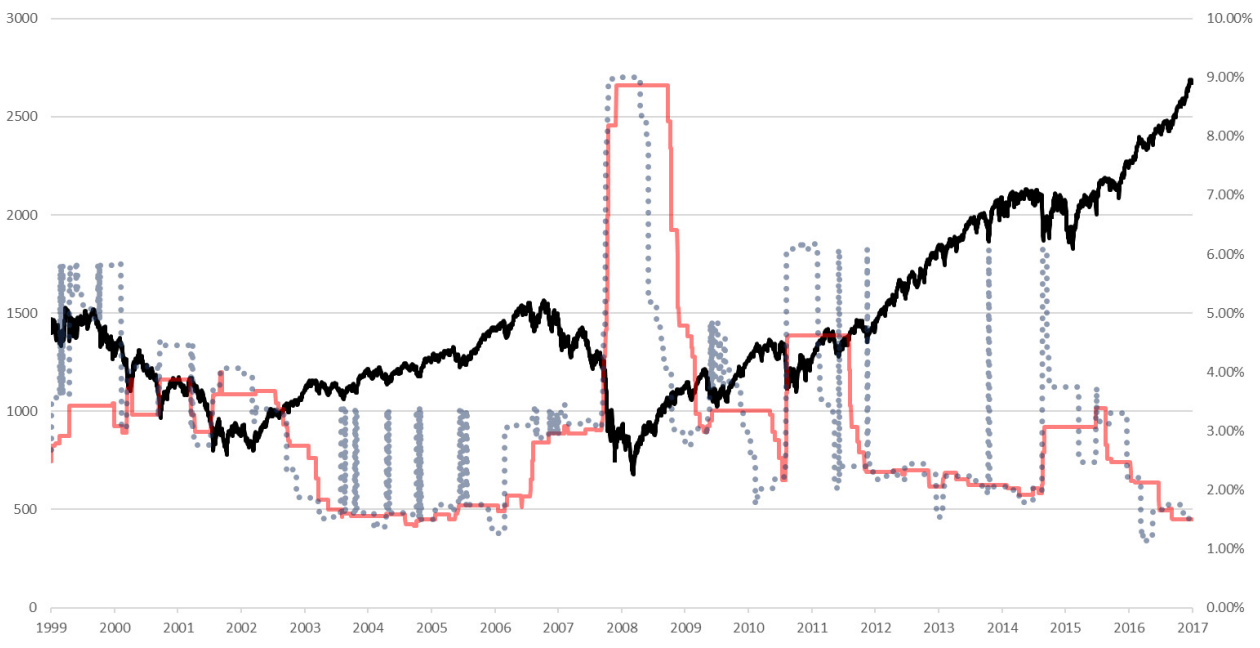

(a)
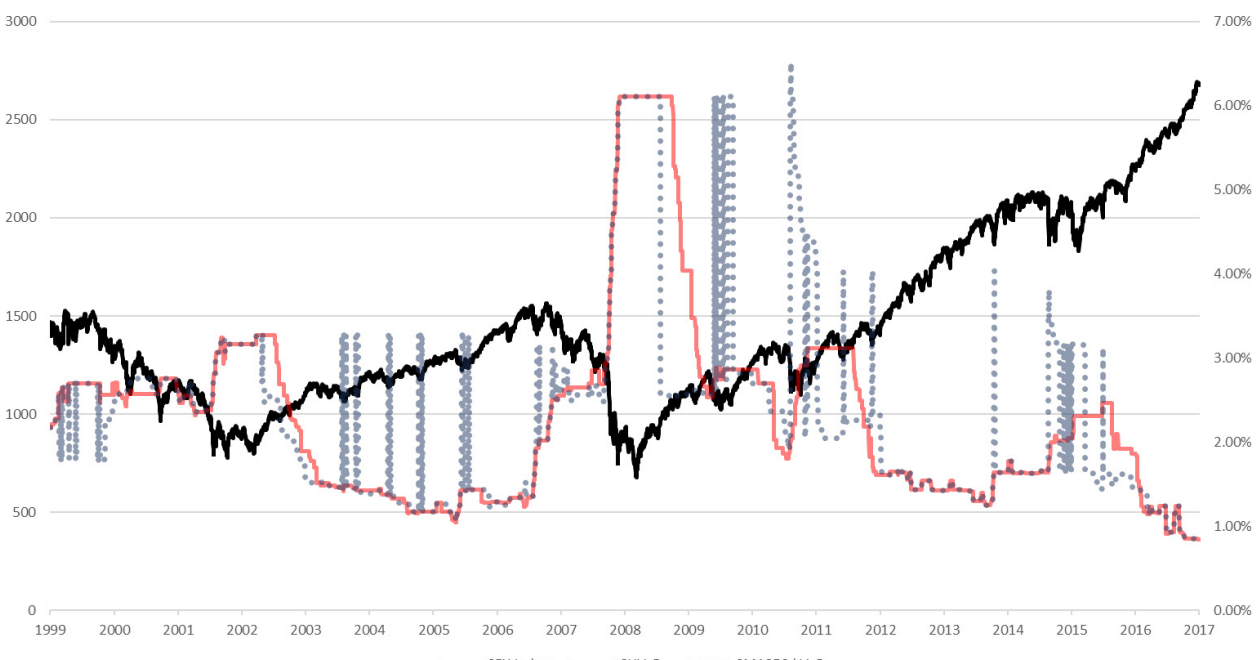

(b)

Fig. 3. SPX index performance, CHVaR models and the $\mathrm{SMA}_{250} \mathrm{HVaR}$ model. (a) SPX index performance, CHVaR models and the $\mathrm{SMA}_{250} \mathrm{HVaR}$ model at the $99 \%$ confidence level and (b) SPX index performance, CHVaR models and the SMA250 HVaR model at the $97.5 \%$ confidence level.

further improved because the approach is based on two individual sub-procedures, data filtering and VaR estimation, and that makes it extremely flexible, because:

(a) for the filtering criterion we can use the same, similar or other filtering criteria. In our study, we use the trading rules as a criterion, but other criteria such as kurtosis (Hull \& White 1998), volatility (Vasileiou 2017), etc. values may be 
used/applied. However, even when the filtering criteria are based solely on the TA rules there are many choices:

(i) several other trading rules, e.g. MACD, RSI, etc. could be used instead of SMA,

(ii) optimization procedures could be applied e.g. to determine which is the optimal l-lag. and

(iii) more advanced TA modeling approaches (e.g. Exponentially Weighted Moving Average GARCH, Kwon \& Kish 2002, Coakley et al. 2016) may be applied and

(b) regarding VaR modeling, several VaR models other than the CHVaR we present in this study can be applied: DNVaR, MCVaR, GARCH family models, etc. for the VaR estimations using filtered data and

(c) an optimization procedure could be applied regarding the optimal number of data inputs that could be used. For example, in our study we use the last 250 observations because this is the minimum number of observations that is required by the legislative framework. The results of the $\mathrm{SMA}_{250} \mathrm{TA} \mathrm{HVaR}$ models when 125 observations (data inputs) are applied are presented in Table 5. The newly suggested model's VaR estimations are more accurate than before (compare $\mathrm{SMA}_{250}$ TA HVaR results in Tables 3 and 5), and as the results show the TA HVaR models fail to meet the four overshootings rule only once.

It is worth mentioning a deficiency of the legislative framework that was revealed in Table 5. The law has a minimum requirement of 250-data inputs for the VaR estimation. Even in theory this restriction is false when we examine the legislative framework. The law does not dictate which model should be used to assess risk, but the guidelines require periodical evaluation of each model based on the accuracy of its estimations. So, what do restrictions on data input really offer? The reply to this question could be that this restriction does not offer something, because we often evaluate the model (every month), so when a model is inaccurate (more than four overshootings per year) it should change. The change of the number of the data inputs is considered as a sufficient revision, provided that the new model does not violate the four-overshooting rule.

In practice, if we use 250 observations and the model is inaccurate, we can increase the number of data inputs in order to present fewer overshootings. ${ }^{r}$ However, if a model is more accurate when fewer than 250 observations are used we cannot use it as a basic model (unless extreme market conditions emerge and justify the change for the specific period (CESR 2010, p. 31). As we present in Table 5, we do not need the restriction on the number of data inputs, because if the problem is the observation period the modification is very easy to be applied. Therefore, the specific restriction does not offer something, in contrast, it may reduce the model's

\footnotetext{
${ }^{r}$ We may increase the number of observations in order to include a crisis period, thus making the VaR more conservative with fewer overshootings (Vasileiou 2016).
} 
Table 5. $\mathrm{SMA}_{250}$ TA HVaR model using 125 observations at the $99 \%$ confidence level.

\begin{tabular}{|c|c|}
\hline & $\mathrm{SMA}_{250} \mathrm{TA} \mathrm{HVaR}$ \\
\hline \multicolumn{2}{|c|}{ Panel A. Descriptive statistics of the CHVaR and the $\mathrm{SMA}_{250}$ TA HVaR model for the SPX $(2000-2017)$} \\
\hline Violations & 38 \\
\hline Violations (\%) & $0.84 \%$ \\
\hline Mean & $-3.15 \%$ \\
\hline SD & $1.64 \%$ \\
\hline Min & $-9.01 \%$ \\
\hline Max & $-1.14 \%$ \\
\hline MSE & $0.142 \%$ \\
\hline Deviations when overshootings are observed & $-0.805 \%$ \\
\hline \multicolumn{2}{|c|}{ Panel B. Backtesting results of the CHVaR and the $\mathrm{SMA}_{250}$ TA HVaR model for the SPX (2000-2017) } \\
\hline 2000 & 2 \\
\hline 2001 & 2 \\
\hline 2002 & 3 \\
\hline 2003 & 2 \\
\hline 2004 & 2 \\
\hline 2005 & 1 \\
\hline 2006 & 1 \\
\hline 2007 & 2 \\
\hline 2008 & 5 \\
\hline 2009 & 0 \\
\hline 2010 & 3 \\
\hline 2011 & 4 \\
\hline 2012 & 0 \\
\hline 2013 & 2 \\
\hline 2014 & 3 \\
\hline 2015 & 3 \\
\hline 2016 & 1 \\
\hline 2017 & 2 \\
\hline Sum of violations & 38 \\
\hline
\end{tabular}

accuracy when a model works better with an observation period shorter than 250 days (see $\mathrm{SMA}_{250}$ TA HVaR results in Tables 3 and 5).

Therefore, as we presented in the previous section the TA adjustments can easily be applied to the CHVaR model and significantly improve the VaR estimations. However, as we present in this section scholars/risk analysts that are more familiar with optimization and other programming techniques can further improve the VaR estimations accuracy.

\section{Conclusions}

In the last decades, there has been an increased number of financial crises, even though econometric and computational finance has presented significant advances. We assume that this contraposition exists because the contemporary advanced models are incomprehensible to nonmathematicians (Fama 1995). An explanation 
for the modeling complexity was given by Ross (1993), who mentions that during the estimation process contemporary models seem to torture the data to confess the crime (accurate VaR estimations in our case). Therefore, in many cases advanced econometric VaR models are not usually applied in the financial industry due to their complexity. In order to bridge the gap between financial econometrics and the financial industry we try to introduce an easy to apply VaR model which presents accurate and representative (nonprocyclical) VaR estimations.

We examine the CHVaR model, which is very easy to be applied, and we provide empirical evidence that it can lead to accurate VaR estimations when the data input is representative of the examined financial conditions. In this study, we filter the data inputs in growth and recession regimes, and we use the suggestions provided by the SMA trading rules as a filtering criterion. When the trading rules suggest that the financial conditions are positive, we use as inputs the observations from the dataset which include daily returns from positive financial trend periods (and vice versa for the recession periods). This way we estimate the $\mathrm{VaR}$ using the last $x$-observations which reveal how the market reacted when the financial conditions were similar to the currently examined. By using this filtering procedure, we try to resolve the Garbage In, Garbage Out issue.

Simultaneously, the suggested approach does not torture the data using complex equations, but it filters the data inputs in order to select and use for the estimations only the most representative past observations, thus achieving more accurate results than the conventional model(s). Therefore, when the market trend changes, a different $\mathrm{VaR}$ regime appears, in order to capture the market trend changes faster than the CHVaR model. This market regime changes resolves the CHVaR model's drawback of inaccurate and procyclical VaR estimations when the financial trend changes (Hendricks 1996, Brooks \& Persand 2009, Vasileiou \& Pantos 2018), and shows that it is not always the models that are inaccurate, but in many cases it is the inappropriate data that make the models appear unsuccessful. Moreover, the TA HVaR model shows a new approach for VaR estimations that are more accurate than the conventional, while the modeling is not as complex as the advanced models, presenting a new view/solution for the accuracy-complexity trade-off in VaR.

Examining the US stock market, we provide empirical evidence that the new approach (TA HVaR) using the SMA data filtering procedure, improves the CHVaR models VaR estimations when the legislative guidelines of minimum 250 observations are followed, because it improves their representativeness of the real financial risk, which in turn reduces the procyclicality of VaR estimations (Table 4). This way when the trading signals accurately confirm/forecast the market trend changes, they could be beneficial in the VaR field of study, regardless of whether or not their profits outperform the BnH strategy.

The specific approach is so flexible that with the appropriate adjustments it could be applied to any market where the TA rules are effective. Moreover, it may be presented here in its simplest form, but mathematically oriented scholars/ practitioners can also adopt this approach and introduce more complex modifications 
to the examined model and/or combine the filtering approach (inputs stage) with more advanced than the CHVaR model (e.g. a GARCH family model). Additionally, the TA HVaR approach could be useful for the portfolio managers who adopt TA strategies in order to estimate the reward to VaR ratio (Alexander \& Baptista 2003), and/or the risk of the Trend Following funds, with the appropriate adjustments.

Beyond the modeling part, we present some suggestions for improving current legislation on risk assessment. The legislative framework examines only the number of overshootings per year at the $99 \%$ c.l., which does not adequately protect the financial system against procyclicality. The adoption of advanced backtesting procedures offers a solution, but, as in the case of the mathematically advanced VaR models, their application remains difficult because they are complex and require specialized knowledge. In order to encourage the use of accurate VaR models we suggested an additional backtest which is based on the scientifically accepted binomial Kupiec test (1995) test at the $97.5 \%$ c.l. and which enables us to test whether the VaR estimations over- and under-estimate the risk. This way the legislative framework will force financial institutions and investment companies to adopt accurate and representative $\mathrm{VaR}$ models, to avoid/reduce the procyclicality and hence to effectively protect the financial system. Finally, we show (Table 5) that restrictions on the number of observations in the form of the mandatory minimum of 250 observations may be an obstacle to more accurate VaR estimations. This restriction does not contribute towards financial stability; therefore, it should be revised or eliminated.

\section{References}

T. Adrian \& H. S. Shin (2013) Procyclical leverage and value-at-risk, The Review of Financial Studies 27 (2), 373-403.

G. J. Alexander \& A. M. Baptista (2003) Portfolio performance evaluation using value at risk, Journal of Portfolio Management 29 (4), 93-102.

F. Allen, M. K. Chui \& A. Maddaloni (2004) Financial systems in Europe, the USA, and ASIA, Oxford Review of Economic Policy 20 (4), 490-508.

T. Angelidis, A. Benos \& S. Degiannakis (2004) The use of GARCH models in VaR estimation, Statistical Methodology 1, 105-128.

J. B. Ang (2008) A survey of recent developments in the literature of finance and growth, Journal of Economic Surveys 22 (3), 536-576.

A. Assaf (2009) Extreme observations and risk assessment in the equity markets of MENA region: tail measures and value-at-risk, International Review of Financial Analysis 18, 109-116, doi: 10.1016/j.irfa.2009.03.007.

Basel Committee on Banking Supervision (1995) An Internal Model-Based Approach to Market Risk Capital Requirements. Basel, Switzerland, https://www.bis.org/publ/ bcbsc224.pdf.

Basel Committee on Banking Supervision (1996) Supervisory Framework for the Use of Backtesting in Conjunction with the Internal Models Approach to Market Risk Capital Requirements. Basel, Switzerland. 
Basel Committee on Banking Supervision (2006) International Convergence of Capital Measurement and Capital Standards. A Revised Framework. Comprehensive Version. Basel, Switzerland: Bank for International Settlements.

T. Beck, R. Levine \& N. Loayza (2000) Finance and the sources of growth, Journal of Financial Economics 58 (1-2), 261-300.

M. Billio \& L. Pelizzon (2000) Value-at-risk: A multivariate switching regime approach, Journal of Empirical Finance 7 (5), 531-554.

W. Brock, J. Lakonishock \& B. LeBaron (1992) Simple technical trading rules and the stochastic properties of stock returns, Journal of Finance 47 (5), 1731-1764.

C. Brooks \& G. Persand (2000) Value-at-risk and market crashes, Journal of Risk 2, 5-26, doi: 10.21314/JOR.2000.029.

J. Coakley, M. Marzano \& J. Nankervis (2016) How profitable are FX technical trading rules? International Review of Financial Analysis 45, 273-282.

Committee of European Securities Regulators (CESR, 2010) CESR's Guidelines on Risk Measurement and the Calculation of Global Exposure and Counterparty Risk for UCITS. CESR/10-788.

P. F. Diamandis, A. A. Drakos, G. P. Kouretas \& L. Zarangas (2011) Value-at-risk for long and short trading positions: Evidence from developed and emerging equity markets, International Review of Financial Analysis 20 (3), 165-176.

R. Engle (2004) Risk and volatility: Econometric models and financial practice, The American Economic Review 94 (3), 405-420.

E. Fama and E. Blume (1966) Filter rules and stock market trading, Journal of Business 39, 226-241.

E. F. Fama (1995) Random walks in stock market prices, Financial Analysts Journal 51 (1), $75-80$.

D. Hendricks (1996) Evaluation of value-at-risk models using historical data (digest summary), Economic Policy Review Federal Reserve Bank of New York 2 (1), 39-67.

J. Hull \& A. White (1998) Value at risk when daily changes in market variables are not normally distributed, Journal of Derivatives 5, 9-19.

J. Á. Jiménez-Martín, M. McAleer \& T. Pérez-Amaral (2009) The ten commandments for managing value at risk under the basel II accord, Journal of Economic Surveys 23 (5), 850-855.

P. H. Kupiec (1995) Techniques for verifying the accuracy of risk measurement models, The Journal of Derivatives 3 (2), 73-84.

K. Y. Kwon \& R. J. Kish (2002) Technical trading strategies and return predictability: NYSE, Applied Financial Economics 12 (9), 639-653.

R. Levine (1997) Financial development and economic growth: Views and agenda, Journal of Economic Literature 35 (2), 688-726.

T. J. Linsmeier \& N. D. Pearson (2000) Value at risk, Financial Analysts Journal 56 (2), $47-67$.

D. P. Louzis, S. Xanthopoulos-Sisinis \& A. P. Refenes (2014) Realized volatility models and alternative Value-at-Risk prediction strategies, Economic Modelling 40, 101-116.

S. Mabrouk \& S. Saadi (2012) Parametric value-at-risk analysis: Evidence from stock indices, The Quarterly Review of Economics and Finance 52 (3), 305-321.

A. M. Moussa, J. S. Kamdem \& M. Terraza (2014) Fuzzy value-at-risk and expected shortfall for portfolios with heavy-tailed returns, Economic Modelling 39, 247-256.

R. T. F. Nazário, J. L. e Silva, V. A. Sobreiro \& H. Kimura (2017) A literature review of technical analysis on stock markets, The Quarterly Review of Economics and Finance.

S. N. Neftci (1991). Naive trading rules in financial markets and wiener-kolmogorov prediction theory: A study of "technical analysis". Journal of Business, 549-571. 
O. Peia \& K. Roszbach (2015) Finance and growth: Time series evidence on causality, Journal of Financial Stability 19, 105-118.

M. Pritsker (1997) Evaluating value at risk methodologies: Accuracy versus computational time, Journal of Financial Services Research 12 (2-3), 201-242.

C. M. Reinhart \& K. S. Rogoff (2008) Is the 2007 US sub-prime financial crisis so different? An international historical comparison, American Economic Review 98 (2) 339-344.

S. A. Ross (1993) Is beta useful? In: AIMR Conference Proceedings, Vol. 1993, No. 6, pp. 11-15 (Association for Investment Management and Research).

S. Stavroyiannis, I. Makris, V. Nikolaidis \& L. Zarangas (2012) Econometric modeling and value-at-risk using the Pearson type-IV distribution, International Review of Financial Analysis 22, 10-17.

R. Sweeney (1988) Some filter rule tests: Methods and results, Journal of Financial and Quantitative Analysis 23 (3), 285-300.

N. Ülkü \& E. Prodan (2013) Drivers of technical trend-following rules' profitability in world stock markets, International Review of Financial Analysis 30, 214-229.

E. Vasileiou (2016) Overview of the Greek value at risk (VaR) legislation framework, Journal of Financial Regulation and Compliance 24 (2), 213-226.

E. Vasileiou (2017) Value at Risk (VaR) historical approach: Could it be more historical and representative of the real financial risk environment? Theoretical Economics Letters $\mathbf{7}$, 951-974.

E. Vasileiou \& T. Pantos (2018) What do the value at risk measure and the respective legislative framework really offer to financial stability? Critical views and procyclicality, European Journal of Economics and Economic Policies: Intervention (forthcoming).

A. Yi-Hou Huang \& T. W. Tseng (2009) Forecast of value at risk for equity indices: An analysis from developed and emerging markets, The Journal of Risk Finance 10 (4) 393-409.

P. Youngman (2009) Procyclicality and value at risk. In: Bank of Canada Financial System Review.

H. G. Zhang, C. W. Su, Y. Song, S. Qiu, R. Xiao \& F. Su (2017). Calculating Value-at-Risk for high-dimensional time series using a nonlinear random mapping model, Economic Modelling 67, 355-367. 